\title{
Eckhoff's problem on convex sets in the plane
}

\author{
Adam S. Jobson \\ Department of Mathematics \\ University of Louisville \\ Louisville, KY 40292, U.S.A. \\ adam.jobson@louisville.edu
}

\author{
André E. Kézdy \\ Department of Mathematics \\ University of Louisville \\ Louisville, KY 40292, U.S.A. \\ kezdy@louisville.edu
}

\author{
Jenő Lehel \\ Alfréd Rényi Institue of Mathematics \\ Budapest, Hungary \\ and \\ Department of Mathematics \\ University of Louisville \\ Louisville, KY 40292, U.S.A. \\ lehelj@renyi.hu
}

Submitted: Oct 28, 2020; Accepted: Jun 11, 2021; Published: Aug 27, 2021

(C) The authors. Released under the CC BY-ND license (International 4.0).

\begin{abstract}
Eckhoff proposed a combinatorial version of the classical Hadwiger-Debrunner $(p, q)$-problems as follows. Let $\mathcal{F}$ be a finite family of convex sets in the plane and let $m \geqslant 1$ be an integer. If among every $\left(\begin{array}{c}m+2 \\ 2\end{array}\right)$ members of $\mathcal{F}$ all but at most $m-1$ members have a common point, then there is a common point for all but at most $m-1$ members of $\mathcal{F}$. The claim is an extension of Helly's theorem $(m=1)$. The case $m=2$ was verified by Nadler and by Perles. Here we show that Eckhoff 's conjecture follows from an old conjecture due to Szemerédi and Petruska concerning 3-uniform hypergraphs. This conjecture is still open in general; its solution for a few special cases answers Eckhoff's problem for $m=3,4$. A new proof for the case $m=2$ is also presented.
\end{abstract}

Mathematics Subject Classifications: 52A10, 52A35, 05C62, 05D05, 05D15, $05 \mathrm{C} 65$

\section{Introduction}

The subject of this note is a combinatorial version of the classical Hadwiger-Debrunner $(p, q)$-problems proposed by Eckhoff [2] (see also [1]). A family $\mathcal{F}$ of convex sets in the 
plane has the $\Delta(m)$-property if $\mathcal{F}$ has at least $|\mathcal{F}|-m+1$ sets with non-empty intersection. We restate Eckhoff's conjecture using this notation.

Problem 1. (Eckhoff [2, Problem 6]) Let $m \geqslant 1, k=\left(\begin{array}{c}m+2 \\ 2\end{array}\right)$ be integers, and let $\mathcal{F}$ be a family of at least $k$ convex sets in $\mathbb{R}^{2}$. If every $k$ members of $\mathcal{F}$ has the $\Delta(m)$-property, then $\mathcal{F}$ also has the $\Delta(m)$-property.

Due to Helly's theorem [5], Problem 1 has a positive answer for $m=1$. The claim was verified also for $m=2$ by Nadler [8] and by Perles [9]. In this note we show that Eckhoff's conjecture follows from an old conjecture due to Szemerédi and Petruska [10] on 3-uniform hypergraphs.

In Section 2, Problem 1 is restated first (Problem 2) in terms of 2-representable 3uniform hypergraphs. The Szemerédi-Petruska conjecture, as reformulated by Lehel and Tuza [11, Problem 18.(a)] states that $\left(\begin{array}{c}m+2 \\ 2\end{array}\right)$ is the maximum order of a 3 -uniform $\tau$-critical hypergraph with transversal number $m$. Thus Eckhoff's conjecture becomes equivalent to a particular instance of a general extremal hypergraph problem (Theorem 6). The Szemerédi-Petruska conjecture is verified for $m=2,3,4$ (see [7]) using the concept of 3uniform $\tau$-critical hypergraphs, cross-intersecting set-pair systems, and $\tau$-critical graphs; this solves Eckhoff's problem for $m=3$, 4 , with a new proof for $m=2$ (Corollary 7).

Eckhoff made the remark that the value of $k$ in Problem 1 is not expected to be tight. Examples in Section 5 show that $k=\left(\begin{array}{c}m+2 \\ 2\end{array}\right)$ cannot be lowered for $m=2,3$, but it is not optimal for $m=4$.

\section{Convex hypergraphs}

Given a family $\mathcal{F}$ of convex sets in $\mathbb{R}^{2}$, let $H$ be the 3-uniform intersection hypergraph defined by vertex set $V(H)=\{F: F \in \mathcal{F}\}$ and edge set $E(H)=\{\{A, B, C\}: A, B, C \in$ $\mathcal{F}$ and $A \cap B \cap C \neq \varnothing\}$.

A 3 -uniform hypergraph $H$, that is the intersection hypergraph of some family $\mathcal{F}$ of planar convex sets is called a 2-representable or convex hypergraph. Observe that a $k$-clique $N \subset V$ of the intersection hypergraph indicates that the $k$ convex sets of $\mathcal{F}$ corresponding to the vertices of $N$ have a common point in the plane, due to Helly's theorem. Eckhoff's problem is stated next in terms of convex hypergraphs.

Problem 2. Let $m \geqslant 1$ and $n \geqslant\left(\begin{array}{c}m+2 \\ 2\end{array}\right)$ be integers, and let $H$ be a 2-representable 3-uniform hypergraph of order $n$. If $\omega(H[X]) \geqslant\left(\begin{array}{c}m+2 \\ 2\end{array}\right)-m+1$, for every $X \subseteq V$, $|X|=\left(\begin{array}{c}m+2 \\ 2\end{array}\right)$, then $\omega(H) \geqslant n-m+1$.

Observe that by Helly's theorem, a family $\mathcal{F}$ of $k$ convex sets in $\mathbb{R}^{2}$ has the $\Delta(m)$ property if and only if the 3-uniform intersection hypergraph $H$ defined by $\mathcal{F}$ has clique number $\omega(H) \geqslant k-m+1$. This implies the equivalence of Problem 1 and Problem 2 . 


\section{$3 \tau$-critical 3-uniform hypergraphs}

Let $H=(V, E)$ be an $r$-uniform hypergraph. For $X \subset V$ define the subhypergraph $H[X]$ on vertex set $X$ with all those edges in $E$ that are contained by $X$. For $e \in E$, denote $H-e$ the partial hypergraph with vertex set $V$ and edge set $E \backslash\{e\}$. Let $\widehat{H}=(V, \widehat{E})$ be the $r$-uniform hypergraph obtained as the complement of $H$ with $\widehat{E}$ containing all $r$-element subsets of $V$ not in $E$.

The transversal number of a hypergraph $H$ is defined by

$$
\tau(H)=\min \{|T|: T \subset V, e \cap T \neq \varnothing, \text { for each } e \in E\} .
$$

A hypergraph $H$ is $\tau$-critical if it has no isolated vertex $\left(\bigcup_{e \in E} e=V\right)$ and $\tau(H-e)=$ $\tau(H)-1$ for every $e \in E$. Let $v_{\max }(r, t)$ be the maximum order of an $r$-uniform $\tau$-critical hypergraph $H$ with $\tau(H)=t$. This function was introduced and investigated by Gyárfás et al. [4] and by Tuza [11, Section 4.2].

Denote $\omega(H)$ the clique number of $H$ defined as the maximum cardinality of a subset $N \subset V$ such that every $r$-element set of $N$ belongs to $E$.

\section{Lemma 3.}

(a) If $\widehat{H}$ is a $\tau$-critical $r$-uniform hypergaph, then the maximum cliques of $H$ have no common vertex.

(b) If the maximum cliques of an r-uniform hypergaph $H$ have no common vertex, then $|V| \leqslant v_{\text {max }}(r, t)$, where $t=\tau(\widehat{H})$.

Proof. Notice that $N \subset V$ is a minimum cardinality transversal of $\widehat{H}$ if and only if $T=V \backslash N$ is the vertex set of a maximum cardinality clique of $H$.

(a) By definition, $\widehat{H}$ has no isolated vertex. Furthermore, for every $x \in V$ and $e \in \widehat{E}$, $x \in e$, we have $\tau(\widehat{H}[V \backslash\{x\}]) \leqslant \tau(\widehat{H}-e)=\tau(\widehat{H})-1$. Then the union of $\{x\}$ with a $(\tau(\widehat{H})-1)$-element transversal of $\widehat{H}[V \backslash\{x\}]$ forms a minimum transversal of $\widehat{H}$. Therefore, every $x \in V$ belongs to some minimum transversal of $\widehat{H}$. Equivalently, the complements of the minimum transversals of $\widehat{H}$, the maximum cliques of $H$, have no common vertex.

(b) Because the maximum cliques in $H$ have no common vertex, the union of their complement in $V$, that is, the union of the $t$-element transversals of $\widehat{H}$, is equal to $V$. Let $H^{\prime}$ be a $\tau$-critical partial hypergraph of $\widehat{H}$ with vertex $V^{\prime}$ and $\tau\left(H^{\prime}\right)=t$. We claim that $\left|V^{\prime}\right|=|V|$.

Because every vertex $x \in V \backslash V^{\prime}$ belongs to some $t$-element transversal $T$ of $\widehat{H}$, the set $T \backslash\{x\}$ is a $(t-1)$-element transversal for all edges of $\widehat{H}$ not containing $x$; hence $\tau\left(H^{\prime}\right)<t$, a contradiction. Thus $|V|=\left|V^{\prime}\right| \leqslant v_{\max }(r, t)$ follows.

Recall that $v_{\max }(3, m)$ is the maximum order of a 3 -uniform $\tau$-critical hypergraph $H$ with $\tau(H)=m$. The conjecture that $v_{\max }(3, m)=\left(\begin{array}{c}m+2 \\ 2\end{array}\right)$ for every $m$ [11, Problem 18.(a)] was verified only for a few small values of $m$ : 
Proposition 4 ([7]). Let $m=2,3$, or 4 , and $n>m$. If $H$ is a 3-uniform hypergraph of order $n$ with clique number $\omega(H)=n-m=k \geqslant 3$ and the intersection of the $k$-cliques of $H$ is empty, then $n \leqslant\left(\begin{array}{c}m+2 \\ 2\end{array}\right)$.

Corollary 5. $v_{\max }(3, m)=\left(\begin{array}{c}m+2 \\ 2\end{array}\right)$, for $m=2,3$ and 4 .

Proof. For every $m \geqslant 1$, a 3-uniform $\tau$-critical hypergraph of order $n=\left(\begin{array}{c}m+1 \\ 2\end{array}\right)+m+1$ with transversal number $m$ is obtained from the complete graph $K_{m+1}$ by extending each edge with one vertex using additional distinct vertices. This construction implies $v_{\text {max }}(3, m) \geqslant\left(\begin{array}{c}m+2 \\ 2\end{array}\right)$.

Let $\widehat{H}$ be a $\tau$-critical 3-uniform hypergraph with $\tau(\widehat{H})=m$ and $|V|=v_{\max }(3, m)$. By Lemma 3(a) and by applying Proposition 4, we obtain $|V|=v_{\max }(3, m) \leqslant\left(\begin{array}{c}m+2 \\ 2\end{array}\right)$, $m=2,3,4$. Thus $v_{\max }(3, m)=\left(\begin{array}{c}m+2 \\ 2\end{array}\right)$ follows for $m=2,3$ and 4 .

\section{$4 \quad$ Eckhoff's problem and $\tau$-critical hypergraphs}

Eckhoff's problem relates to the hypergraph extremal problem of determining $v_{\max }(3, m)$ as is shown by the next theorem.

Theorem 6. For $m \geqslant 1$ and $n \geqslant k \geqslant v_{\max }(3, m)$, let $\mathcal{F}$ be a family of $n$ convex sets in $\mathbb{R}^{2}$. If every $k$ members of $\mathcal{F}$ have the $\Delta(m)$-property, then $\mathcal{F}$ has the $\Delta(m)$-property.

Proof. Assume that the claim is not true. Let $H_{0}$ be a 3-uniform convex hypergraph of minimum order $n_{0}$ such that $\omega\left(H_{0}\right) \leqslant n_{0}-m$, but $\omega\left(H_{0}[X]\right) \geqslant k-m+1$, for every $X \subset V_{0},|X|=k$. Notice that the definition of $H_{0}$ implies $n_{0}>k$; furthermore, since $n_{0}$ is minimal, $\omega\left(H_{0}\right)=n_{0}-m$.

We claim that the intersection of the maximum cliques of $H_{0}$ is empty. If $x \in V_{0}$ was a common vertex of all maximum cliques, then $H^{\prime}=H_{0}\left[V_{0} \backslash\{x\}\right]$ has order $n^{\prime}=n_{0}-1$, and for its clique number we have $\omega\left(H^{\prime}\right)=\omega\left(H_{0}\right)-1=n_{0}-m-1=n^{\prime}-m$. At the same time, $\omega\left(H^{\prime}[X]\right) \geqslant k-m+1$, for every $k$-element subset $X \subset V_{0} \backslash\{x\}$. Hence $H^{\prime}$ is a counterexample of order $n^{\prime}$, contradicting the minimality of $n_{0}$. Therefore, the maximum cliques of $H_{0}$ have no common vertex, and because $\tau\left(\widehat{H_{0}}\right)=n_{0}-\omega\left(H_{0}\right)=m$, Lemma 3 implies $k<n_{0} \leqslant v_{\max }(3, m) \leqslant k$, a contradiction.

As an immediate corollary of Theorem 6 and Proposition 5 we obtain an extensions of Helly's theorem together with a combinatorial proof for the case $m=2$ (verified earlier by Nadler [8] and by Perles [9]).

Corollary 7. Let $1 \leqslant m \leqslant 4, k=\left(\begin{array}{c}m+2 \\ 2\end{array}\right)$, and let $\mathcal{F}$ be a family of at least $k$ convex sets in $\mathbb{R}^{2}$. If every $k$ members of $\mathcal{F}$ has the $\Delta(m)$-property, then $\mathcal{F}$ also has the $\Delta(m)$ property. 


\section{Concluding remarks}

5.1 The best known general bound $v_{\max }(3, m) \leqslant \frac{3}{4} m^{2}+m+1$ is obtained by Tuza ${ }^{1}$ using the machinery of $\tau$-critical hypergraphs. This bound combined with Theorem 6 yields the following finiteness result on Eckhoff's problem, for every $m$.

Corollary 8. Let $\mathcal{F}$ be a family of at least $k \geqslant \frac{3}{4} m^{2}+m+1$ convex sets in $\mathbb{R}^{2}$. If every $k$ members of $\mathcal{F}$ has the $\Delta(m)$-property, then $\mathcal{F}$ also has the $\Delta(m)$-property.

5.2 In Corollary 7 the value of $k$ is optimal (the smallest possible) if there is a family of $n \geqslant k$ convex sets in $\mathbb{R}^{2}$ such that every $k-1$ members of $\mathcal{F}$ satisfy the $\Delta(m)$-property, but $\mathcal{F}$ fails it. It was proved by Nadler [8] that $k=\left(\begin{array}{c}m+2 \\ 2\end{array}\right)$ is optimal for $m=2$, but as noted by Eckhoff [1], it is 'somewhat unlikely' that it is optimal for every $m$. We address optimality for $m=2,3,4$ by defining a family $\mathcal{F}_{m}$ of convex sets as follows.

$\mathcal{F}_{2}: m=2, k=6$. Let $\mathcal{F}_{2}$ be the family of $n=6$ line segments, taken each side of the triangle $T=(p, q, r)$ twice. Then any vertex of $T$ covers only $4=n-m$ members of $\mathcal{F}_{2}$; meanwhile, when removing a copy of one side, say $\overline{q r}$, vertex $p$ covers $(k-1)-(m-1)=4$ members.

$\mathcal{F}_{3}: m=3, k=10$. Let $p_{0}, p_{1}, p_{2}, p_{3}, p_{4} \in \mathbb{R}^{2}$ be the vertices of a regular pentagon $P$, and let $\mathcal{F}_{3}$ be the family of $n=10$ convex sets: the five triangles $T_{i}=\left(p_{i}, p_{i+1}, p_{i+2}\right)$ plus the five quadrangles $Q_{i}=\left(p_{i}, p_{i+1}, p_{i+2}, p_{i+3}\right), 0 \leqslant i \leqslant 4$, with $(\bmod 5)$ index arithmetic. Notice that among eight members there are at least three triangles, and among three triangles the intersection of some two is a vertex of $P$, which covers only $7=n-m$ members of $\mathcal{F}_{3}$. On the other hand when removing some member $C$ from $\mathcal{F}_{3}$, any vertex of $P$ not in $C$ covers $(k-1)-(m-1)=7$ members.

$\mathcal{F}_{4}: m=4, k=\left(\begin{array}{c}m+2 \\ 2\end{array}\right)-1=14$. Let $S=\left\{p_{0}, p_{1}, \ldots, p_{7}\right\}$ be the set of vertices of a regular octagon, and let $\mathcal{F}_{4}$ be the family of $n=14$ convex sets defined as follows. Take the eight hexagons determined by the vertex sets $S \backslash\left\{p_{i}, p_{i+1}\right\}, 0 \leqslant i \leqslant 7$, and take the six quadrangles $Q_{i}=\left(p_{i}, p_{i+1}, p_{i+2}, p_{i+3}\right)$, for $i \in\{1,2,3,5,6,7\}$, with $(\bmod 8)$ index arithmetic. Notice that the undefined $Q_{0}, Q_{4}$ do not belong to $\mathcal{F}_{4}$, furthermore, the six quadrangles defined in $\mathcal{F}_{4}$ form three disjoint pairs. Taking one quadrangle from each pair plus the eight hexagons form a subfamily of 11 convex sets with no common point, thus at most $10=n-m$ members of $\mathcal{F}_{4}$ can be covered by one point. On the other hand, three intersecting quadrangles plus seven more hexagons contained in every subfamily $\mathcal{F}_{4} \backslash\{C\}$, that is, $(k-1)-(m-1)=10$ members have a common point $q$ of the plane as it is seen in Fig.1.

Family $\mathcal{F}_{m}$ shows that $k=\left(\begin{array}{c}m+2 \\ 2\end{array}\right)$ is optimal in Corollary 7 for $m=2,3$. Each of $\mathcal{F}_{2}$ and $\mathcal{F}_{3}$ is derived from a 3-uniform hypergraph witnessing $v_{\max }(3, m)=\left(\begin{array}{c}m+2 \\ 2\end{array}\right)$. For $m=4$ the 3 -uniform witness hypergraphs are not 2-representable. This fact was observed by Jobson et al. [6] when a similar method using convex hypergraphs was applied to

\footnotetext{
${ }^{1}$ Personal communication
} 


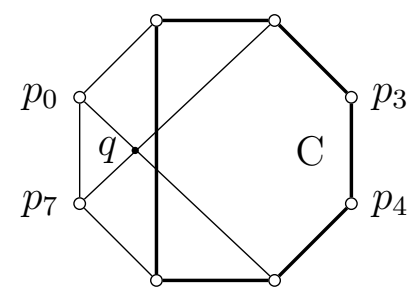

Figure 1: $q$ covers ten members of $\mathcal{F}_{4} \backslash\{C\}$

another geometry problem on convex sets in the plane [6]. Thus the optimum for $m=4$ is less than $\left(\begin{array}{c}4+2 \\ 2\end{array}\right)=15$; and $\mathcal{F}_{4}$ shows that for $m=4$ the optimum value in Corollary 7 is actually $k=\left(\begin{array}{c}4+2 \\ 2\end{array}\right)-1=14$.

5.3 In the light of the discussions above, Eckhoff's problem takes the form of an extremal problem asking for the smallest integer $k(m) \leqslant\left(\begin{array}{c}m+2 \\ 2\end{array}\right)$ such that Theorem 6 remains true when $v_{\max }(3, m)$ is replaced with $k(m)$. The exact values, which we know are $k(1)=3$, $k(2)=6, k(3)=10, k(4)=14$, and we ask the question whether $k(m)=\Omega\left(m^{2}\right)$.

\section{Acknowledgements}

We would like to thank András Gyárfás for calling our attention to Eckhoff's problem, and for his valuable comments to an earlier version of the manuscript.

\section{References}

[1] J. Eckhoff. A survey of the Hadwiger-Debrunner $(p, q)$-problem. in: Discrete and computational geometry, 347-37\%, Algorithms Combin., 25, Springer, Berlin, 2003. https://doi .org/10.1007/978-3-642-55566-4_16.

[2] J. Eckhoff. Problems in discrete geometry. Convexity and discrete geometry including graph theory, 269-273, Springer Proc. Math. Stat., 148, Springer, 2016. https://doi .org/10.1007/978-3-319-28186-5_26.

[3] P. Erdös, and T. Gallai. On the maximal number of vertices representing the edges of a graph. Közl. Magyar Tud. Akad. Mat. Kutató Int. Közl, 6 (1961), 181-203.

[4] A. Gyárfás, J. Lehel, and Zs. Tuza. Upper bound on the order of $\tau$-criti-cal hypergraphs. J. Combin. Theory Ser., B 33 (1982), no. 2, 161-165.

https://doi .org/10.1016/0095-8956(82)90065-X.

[5] E. Helly. Über Mengen konvexer Körper mit gemeinschaftlichen Punkte. Jahresbericht der Deutschen Mathematiker-Vereinigung 32 (1923), 175-176.

[6] A. S. Jobson, A. E. Kézdy, J. Lehel, T. Pervenecki, and G. Tóth. Petruska's question on planar convex sets. Discrete Math., 343 (2020), no. 9, 13pp. 
[7] A. S. Jobson, A. E. Kézdy, and J. Lehel. The Szemerédi-Petruska conjecture for a few small values. Eur. J. Math., May (2021) 8pp.

https://doi.org/10.1007/s40879-021-00466-9

[8] D. Nadler. Minimal 2-fold coverings of $\mathbb{E}^{d}$. Geom. Dedicata, 65 (1997), no 3. 305-312.

[9] M. A. Perles. A Helly type theorem for almost intersecting families. Talk at the Convex Geometry meeting, Oberwolfach. June 1993.

[10] E. Szemerédi, and G. Petruska. On a combinatorial problem I. Studia Sci. Math. Hungar., 7 (1972), 363-374.

[11] Zs.Tuza. Critical hypergraphs and intersecting set-pair systems J. Combin. Theory Ser. B, 39 (1985), no. 2, 134-145.

https://doi.org/10.1016/0095-8956(85)90043-7 